\title{
Fostering collaborative knowledge building by the effective provision of knowledge about the discussion process
}

\section{Santi Caballé ${ }^{* 1}$, Thanasis Daradoumis ${ }^{2}$ and Fatos Xhafa ${ }^{3}$}

$\{1,2\}$ Department of Computer Sciences, Multimedia and Telecommunication, Open University of Catalonia,

Rambla del Poblenou, 156

08018 Barcelona, Spain

Fax: +34 933568822

E-mail: \{scaballe, adaradoumis\}@uoc.edu

\{3\} Department of Languages and Informatics Systems,

Technical University of Catalonia,

Jordi Girona 1-3

08034 Barcelona, Spain

E-mail: fatos@1si.upc.edu

${ }^{*}$ Corresponding author

\begin{abstract}
This paper aims to report an experience of using an innovative CSCL tool to support real, collaborative learning by discussion. We based the success of on-line collaborative discussion on two crucial aspects: first, by providing an adequate dialogue structure and move types that promote meaningful contributions and achieve more effective interaction; second, by extracting relevant knowledge in order to provide learners and tutors with efficient awareness, feedback, and monitoring as regards learners' performance and collaboration. To this end, we employ a sociolinguistic model that builds a structure of the discussion through generic exchange types whereas it identifies a sufficient set of move types that users can use to contribute effectively to an on-line asynchronous discussion activity. Then these move types are used as indicators to analyze learners' interactions and let them and their tutors be aware of their behaviour and performance as well as of the particular skills exhibited during interaction. The ultimate goal is to achieve a more effective support and assessment of the discussion process and to enhance learning. To validate this model, a real virtual learning environment is employed to support collaborative activities based on asynchronous discussion.
\end{abstract}

Keywords: web based distance education; collaborative learning; computer mediated communication; interactive learning environments; architectures for educational technology system;

Biographical notes: Santi Caballe has a Masters and Bachelors in Computer Science from the Open University of Catalonia. Since 2003, he has been an Assistant Professor at the Open University of Catalonia teaching a variety of curses in Computer Science in the areas of Information Systems, Software Engineering and Collaborative Learning. Since early 2006 he has been working as an Associate Professor of the department of Computer 
Author

Science, Multimedia and Telecommunication at the Open University of Catalonia where he completing his $\mathrm{PhD}$. His research focuses on e-learning, software engineering, network technologies, distributed learning, computer-supported collaborative learning, interaction analysis, and grid technologies. His email address is scaballe@uoc.edu.

Thanasis Daradoumis has a PhD in Computer Sciences from the Polytechnic University of Catalonia-Spain, a Masters in Computer Sciences from the University of Illinois, and a Bachelor in Mathematics from the University of Thessaloniki-Greece. Since 1984, he has been an Assistant Professor at several universities in the USA, Greece and Spain, teaching a variety of courses in Mathematics and Computer Science. Since 1998, he has been working as an Associate Professor in the department of Computer Science, Multimedia and Telecommunication at the Open University of Catalonia where he coordinates several online courses as well as the development of teaching materials appropriate for virtual learning. He is a member of the Distributed, Parallel and Collaborative Systems Research Group at the Open University of Catalonia. His research focuses on e-learning, educational and network technologies, distributed learning, ontologies, CSCL, CSCW, interaction analysis, and grid technologies. His email address is adaradoumis@uoc.edu.

Fatos Xhafa received his PhD in Computer Science from the Polytechnic University of Catalonia (Barcelona, Spain) in 1998. He joined the Department of Languages and Informatics Systems of the Polytechnic University of Catalonia as an Assistant Professor in 1996 and is currently Associate Professor of this department. He is member of the ALBCOM Research Group of this department and also is external member of the Distributed, Parallel and Collaborative Research Group of the Open University of Catalonia. His research is supported by several research projects from Spain, European Union and NSF/USA. He has published in leading international journals and conferences. He serves in the editorial board of the International Journal of Computer Supported Collaborative Learning and has served as Co-chair / PC member for many conferences and workshops. His email address is fatos@1si.upc.edu. 
Fostering collaborative knowledge building by the effective provision of knowledge about the discussion process

\section{Introduction}

When developing Computer-Supported Collaborative Learning (CSCL) environments that support online collaborative learning, several issues must be taken into account in order to ensure full support to the online learning activity. One such key issue is interaction management and analysis to support awareness, coaching and evaluation, based on information captured from the actions performed by participants during the collaborative process (Dillenbourg, 1999). The success of CSCL applications depends to a great extent on the capability of such applications to embed information and knowledge extracted from group activity interaction and use it to achieve a more effective group monitoring.

The real context in this study is the virtual learning environment of the Open University of Catalonia (UOC) ${ }^{1}$, which offers full distance education through the Internet. Part of UOC's courses' curricula includes the participation of students in online discussions with the aim of sharing and discussing their ideas. Indeed, the discussion process plays an important social task where participants can think about the activity being performed, collaborate with each other through the exchange of ideas that may arise, propose new resolution mechanisms, as well as justify and refine their own contributions and thus acquire new knowledge (Stahl, 2006).

Schellens \& Valcke (2006) investigated whether collaborative learning in asynchronous discussion groups results in enhancing academic discourse and knowledge construction. Their research work showed that students in the discussion groups were very task-oriented and that higher proportions of high phases of knowledge construction were observed. Moreover, significant increases in the cognitive interaction, taskorientation and higher phases of knowledge construction were detected.

Given the added value of asynchronous discussion groups and the extensive use of online debates, as one of the main elements of the UOC's pedagogical model, it is essential to provide adequate on-line tools to support the whole discussion process, which also includes students' monitoring and evaluation.

Furthermore, an important issue raised in collaborative learning interactions is the change from divergence to shared understanding and to possible construction of knowledge. The point is to understand how collaborative interactions develop over time: whether students raise new issues (ideas) more frequently as they become more familiar with the discussion and discussants, and whether shared knowledge building becomes richer over time, and subsequent evidence that students were able to construct their own understanding based on their interactions with others (Puntambekar, 2006). To this end, our model annotates and examines a variety of elements that contribute to the understanding of the nature of the collaborative interactions, such as the students' passivity, proactivity, reactivity as well as the effectiveness and impact of their contributions to the overall goal of the discussion.

Large amounts of information data are generated from asynchronous discussion which includes complex issues of the collaborative work and learning process (e.g., group well-being (McGrath, 1991) as well as self, peer and group activity evaluation (Daradoumis, Martínez, \& Xhafa, 2006)). On the one hand, quantitative information can be managed by applying a structured process where the users' interactions are tagged with certain indicators according to a collaborative learning conversation skill taxonomy (Soller, 2001) that models the various types of interactions at different levels. Moreover,

\footnotetext{
${ }^{1}$ The UOC is located in Barcelona, Spain, and offers full distance education through the Internet since 1995. The virtual campus supports currently about 40,000 students, lecturers and tutors who are involved in 600 online courses from 23 official degrees and other $\mathrm{PhD}$ and post-graduate programs. The UOC is found at http://www.uoc.edu
} 
Author

typical quantitative indicators about the participants' performance and dynamics (e.g., number of contributions written and read by each participant) are also considered as relevant to model the group functioning and task performance (Daradoumis et al., 2006).

Indeed, quantitative content analysis has been increasingly used to surpass surface level analyses in CSCL (e.g., counting messages) and several content analysis schemes have been employed to analyze transcripts of online asynchronous discussion groups in formal educational settings (De Wever, et. al., 2006). Although this research technique has been often used, standards are not yet established. As a consequence, the empirical base of the validity of the instruments is limited. Several open questions still exist, especially as concerns the unit of analysis and segmentation procedure to be followed (Strijbos, Martens, Prins, \& Jochems, 2006). In a different study, a content analysis scheme has been applied to analyze the way online peer tutoring (conducted by fourthyear students) supports asynchronous discussion groups of first-year students (De Smet, et. al. 2008). This study demonstrates the important role that tutoring plays in online asynchronous discussions, which is taken into account and constitutes a contributing element of our model.

On the other hand, qualitative information is also valuable to complete the laboured task of interaction analysis and evaluation of contributions. Pena-Shaff \& Nicholls (2004) used a mixed approach to analyze student interactions and meaning construction in computer bulletin board discussions. Quantitative analysis was used to examine participation and interaction rates, and qualitative procedures were used to analyze knowledge construction processes and to refine a category system of indicators and descriptors. Results showed that students engaged in a knowledge construction process that was characterized chiefly by clarification, elaboration, and interpretation.

Moreover, a study by Schrire (2006) applies a merging of quantitative analysis within a qualitative methodology to build a model for the analysis of collaborative knowledge building in asynchronous discussion. The model allows examination of the communication from the multiple perspectives of interaction, cognition and discourse analysis. Content analysis of the discourse was done at a number of levels, focusing on the discussion forum itself, the discussion threads, the messages, and the exchanges and moves among the messages. As a result, it was possible to build a scheme for assessing knowledge building in asynchronous discussion groups. The scheme integrates the interactive, cognitive and discourse dimensions in CSCL. Similarly, Bratitsis \& Dimitracopoulou (2006) analyze the quality of group interactions in asynchronous discussion by means of a multi-indicator model based on quantitative aspects of the active and passive behaviour of participants (i.e., number of messages written/replied and read). This may help tutor infer problematic situations occurring during the learning process as well as identify individual behaviour that may influence collaboration, such as passivity and arrogance.

In a more recent work, Hew \& Cheung (2008) report a qualitative study examining the facilitation techniques used by student facilitators to attract their course mates to participate in asynchronous online discussions. This study differs from previous ones in the sense that it does not focus on the role of the tutor as facilitator and promoter of student participation, but it explores peer facilitation. To explore the extent to which student participation in an online discussion forum is successful, the study looks at the depth of discussion threads. Finally, it reports the facilitation techniques that were exhibited by the student facilitators. However, the mere consideration of the depth of discussion threads does not guarantee by itself the quality of the discussion; students' postings can be simply driven by socialization reasons and not directly linked to the development of the learning tasks.

In overall, we believe that there are more evident key discourse elements and aspects that play an important role both for promoting student participation and enhancing group 
Fostering collaborative knowledge building by the effective provision of knowledge about the discussion process

and individual performance, such as, the impact and effectiveness of students' contributions, among others, that we explore in this work. By making these elements explicit, our discussion model accomplishes high students' participation rates and contribution quality in a more natural and effective way. Indeed, our approach goes beyond a mere interaction analysis of asynchronous discussion in the sense that it builds a multi-functional model that fosters knowledge sharing and construction, develops a strong sense of community among students, provides the tutor a powerful tool for students' monitoring, discussion regulation, while it allows peer facilitation through self, peer and group awareness and assessment. To this end, it is important that the system is capable of managing both qualitative and quantitative information and transforming it into useful knowledge for all the implicated parties in an efficient and clear way.

This can be achieved by both the specific assessment of each contribution by the tutor who supervises the discussion and by rich statistical information about student's participation. This statistical data is automatically provided by the system; for instance, statistical data shed light on the students' engagement in the discussion forum or how much interest drew the student's intervention in the form of participation impact, level of passivity, proactivity, reactivity, and so on. The aim is to provide both a deeper understanding of the actual discussion process and a more objective assessment of individual and group activity.

All this information can be easily collected and automatically processed and analyzed by computers as a quantitative and qualitative data source and presented to the participants in order to provide effective information, such as how all participants are actually performing during the discussion and the dynamics of each participant with respect to the group. Consequently, the efficient embedding of all this information and of the extracted knowledge into CSCL applications sets the basis for enhancing support (Puntambekar, 2006), awareness (Gutwin, Stark, \& Greenberg, 1995) and feedback (Zumbach, Hillers, \& Reimann, 2003) to achieve a successful discussion process in collaborative environments. Indeed, the constant and fast processing and presentation of this quantitative and qualitative data as well as their systematic analysis based on principled indicators that measure the type and the degree of group members' participation, may positively impact on participant's motivation, emotional state and problem-solving abilities and as a result enhance the acquisition of knowledge performance (Daradoumis et al., 2006).

The ultimate aim of this work is to extract relevant knowledge of the discussion process from all possible sources (e.g., users' activity, passivity, and effectiveness; participation impact; qualitative assessment, etc.). Note that in this context information refers to quantitative and qualitative data generated by the learning group whereas knowledge refers to the result of the treatment of this information through analysis techniques and interpretation. This knowledge will be fed back and presented to the learning group members and its tutor for awareness, feedback, and scaffolding purposes.

In this paper, all these entire approaches take form by introducing a new collaborative learning tool called Discussion Forum (DF), which was developed to support and enhance the discussion process encountered in many of the UOC's virtual courses in the form of on-line discussions. This system implements many of the approaches described so far and the first results drawn from the real collaborative learning show very promising benefits for students in the learning context of the UOC and in education in general.

The paper is organized as follows. Section 2 presents a theoretical framework whose purpose is to identify and classify the main exchange categories that describes a generic discourse goal during a collaborative discussion process. In Section 3 the main guidelines that conducted the design and development of a Discussion Forum are provided based on 
these principles. The experience and the evaluation results of using this application in a real context are reported in Section 4. Finally, Section 5 concludes by summarizing the main aspects of the contributions presented in this paper.

\section{A model for managing interaction in a discussion process}

This section examines how learning and knowledge building can be supported in the context of an asynchronous collaborative discussion in a virtual learning environment. To this end, a conceptual sociolinguistic framework is defined for modeling dialogue and understanding how learning evolves and how knowledge is constructed during the discussion process. One important issue to consider is the types of interaction that occur and subsequently the knowledge which is manifested in an asynchronous collaborative discussion. This approach aims at identifying the various types of interaction produced and examining how an interaction type is related to the learning that results from it. As a result, this framework allows the study of how knowledge is transformed and becomes common to all discussion members.

In particular, this section examines how the building and distribution of knowledge is manifested in the context of student-student interaction and how it can be studied in a virtual learning environment. This involves the definition of appropriate collaborative learning situations and the distinction of two levels of student interaction, the discourse and the action level.

At the discourse level, the essential element is the interaction among peers (participants need to interact with each other to plan an activity, distribute tasks, explain, clarify, give information and opinions, elicit information, evaluate and contribute to the resolution of problematic issues, and so on). At the action level, task objects (e.g., documents, graphics) are created and manipulated. This approach focuses more at the analysis of the discourse level by seeing discourse as a medium and means through which the building and distribution of cognition is effected.

To satisfy course assessment requirements, discourse contributions also need to be evaluated as effectively as possible. Evaluation of hundreds of contributions in a multimember discussion can be a tedious task for tutors and should be adequately supported. Moreover, self and peer assessment should be also encouraged and facilitated by intuitive means. Then, a dialogue model of asynchronous discourse is to be provided, which is capable of capturing, analyzing and evaluating both the process and the result of the building and distribution of knowledge. This model should be mainly defined in terms of types and structure of student-student interaction.

In particular, the framework proposed in this paper to support this model is based on an integration of several models and methods: the Negotiation Linguistic Exchange Model (Martin, 1992); a model of Discourse Contributions (Clark \& Schaefer, 1989); and, the types of learning actions underlying a participant turn (Self, 1994). The structure of a long interaction is constructed cooperatively by using the exchange as the basic unit for communicating knowledge. Following Martin (1992), three general exchange structure categories are considered: give-information exchange, elicit-information exchange and raise-an-issue exchange, which consist of different types of moves (Schwartz, 1999) and describe a generic discourse goal. More specifically, the goal of the actor who initiates the give-information exchange is to inform his/her partners about a certain situation with the aim to change the partners' mental states. Informing includes moves that explain, give an opinion, describe or remind a situation in different ways. The actor goal of the second exchange is to elicit the partners' state of mind (knowledge, beliefs, attitude, desire or abilities) of a situation which the actor is not aware or certain 
Fostering collaborative knowledge building by the effective provision of knowledge about the discussion process

about. The actor goal of the third exchange is to raise an issue (a problem or question) to be resolved by the participants, which causes to explore their state of mind (knowledge, beliefs, etc.).

According to Martin (1992), there is a move that constitutes the "obligatory move" of the exchange, since it either carries or indicates completion of the discourse goal for which the exchange is initiated. The obligatory move of each of the above exchanges is: the first move of the give-information exchange, the second move of the elicitinformation exchange and the third move of the ascertain-information exchange.

According to Clark \& Schaefer (1989), each move is seen as a contribution to discourse. This means that in a cooperative conversation, contributions are regarded as collective acts performed by the participants working together, resulting in units of conversation - typically turns (moves) - that aim to make a success of the discourse they compose. Yet, not all moves contribute in the same way toward the successful completion of the exchange.

Some moves have a pure contributing function toward the realization of the obligatory move of the exchange. This is the case of the first move of the elicitinformation exchange, as well as of the first and the second moves of the ascertaininformation exchange. In fact, without the presence of those moves, the obligatory move cannot be realized; thus, those moves really contribute toward the realization of the obligatory move. Consequently, it is stated that successful realization of the obligatory move conveys evidence of (initial) success of the exchange (Clark \& Schaefer, 1989).

In contrast, the other moves have a rather supporting function (provide evidence of support) toward the definite completion of the obligatory move and consequently of the exchange. This is the case of the follow-up moves of the three exchanges. Supporting moves are optional, so they may not be realized. In such a case, they convey an implicit support toward the obligatory move, that is, toward the definitive completion of the exchange.

Based on the work of Self (1994), Pilkington (1999), and Soller (2001), partners are involved in a process of realizing a number of learning actions which lead to the completion of the exchange goal. Each move type captures and controls the evolution of the learning action performed by a participant by setting the expectations of the type of learning actions which has to be realized next by the other participants so that the goal set by the initial move be accomplished.

Both the quantity and the quality of the several move types performed are measured by the collaborative effort of the members involved to achieve the discourse goal of an exchange. The term collaborative effort means both the number of contributing and supporting moves issued by a participant, which indicates an active participation (distinguishing between proactive and reactive one) or passive one, and the type and effectiveness of these moves (which indicates the way a participant contributes toward the achievement of the shared discourse goal, as regards knowledge possession and transfer, reasoning capability and positive attitude). The tutor measures move effectiveness by assessing the quality of their content. In addition, peer assessment can be effected to complete the evaluation of each contribution made. The role these moves plays in the exchange as well as the degree of success of that role determine the successful completion of the exchange goal.

Completion of an exchange expresses the mutual beliefs of all participants about the accomplishment of its discourse goal. Moreover, it implies the achievement of a certain degree of knowledge building and distribution among the different participants. This degree can be deduced and measured by exploring the principal interaction indicators proposed by this model. For each participant the model measures: the total number of moves created, his/her participation behavior (proactive, reactive, supportive, or passive), 
Author

the effectiveness and impact that each move has in the discourse and in the achievement of the current discourse goal, as well as the evaluation of the move content and significance by his/her peers and the tutor.

In general, the three types of exchanges represent standard discourse structures for handling information and suggest a certain type of knowledge building, as a result of giving and eliciting information or working out a solution on an issue set up. These discursive structures enable the participants to take turns, share information, exchange views, monitor the work done and plan ahead. Most importantly, they provide a means to represent and operationalize the cognitive product at individual level, that is, the way the reasoning process is distributed over the participants as it is shared in a collaborative discourse.

Consequently, interaction analysis takes into account both the way the interaction is structured and the types of contributions which are explicitly defined and expressed. The analysis of these interactions yields very useful conclusions on aspects such as individual and group working, dynamics, performance and success, which allows the tutor to obtain a global account of the progress of the individual and group work and thus to identify possible conflicts and monitor the whole learning process much better.

A further innovation of this model is that it allows participants to end up an exchange which took several moves to conclude by "replaying" the main contributing move of the exchange. For instance, in a set-up-an-issue exchange, a solution move may not be sufficiently complete and thus has to be further elaborated, corrected or extended. To that end, another participant has the option to provide an amplify-solution move which completes the initial solution. In general, a "replay" move can be used to resume all the changes produced from the initial appearance of an exchange goal to be achieved to its final conclusion and acceptance by all participants. This can be useful both to reinforce the fact that the goal of the exchange has been completed successfully and to explicitly indicate the progress achieved in the participants' process of knowledge building (especially as regards the participant who provided the main contributing move of the exchange).

Finally, the system requires the participant to commit certain action to indicate $\mathrm{s} / \mathrm{he}$ has read a certain contribution, such as send a reply and assent the contribution. The aim is both to provide reliable indicators on the number of contributions read and to promote the discussion's dynamics by increasing the users' interaction with the system.

Next, the definition and measurement of the indicators used to assess participation behavior, knowledge building and performance are discussed.

\subsection{Indicators used to assess participation behavior, knowledge building and performance}

Participation behavior indicators are distinguished into proactive, reactive and supportive (or assentive). Participants are proactive when they take the initiative to open a new exchange of the type give-information, or raise-an-issue. Participants are reactive when they reply to moves such as elicit-information, set-up-an issue/problem, or providesolution. Participants are supportive if they give their assent to previous contributions. In that case, a supporting value is defined which is assigned a default numerical value 1 which means that the move fully supports and recognizes the value, contribution and effectiveness of a previous move it refers to. If several supporting moves refer to a particular move $\mathrm{M}$, it implies a broader consensus about the impact of $\mathrm{M}$, which increases M's impact value to 1 .

Passive participants are considered those who just read others' contributions, as well as the ones who also evaluate the usefulness of these contributions. Passivity becomes an essential indicator for the discussion process' dynamics as it identifies certain important 
Fostering collaborative knowledge building by the effective provision of knowledge about the discussion process

profiles of the participant, such as arrogance (participant who just contributes but does not read the contributions of others) and also promotes reactive attitudes and social grounding skills (Daradoumis et al., 2006) by engaging the participant in the collaborative process.

The impact value is assigned an initial (default) numerical value between 0 and 1 which is modified (increased or decreased) according to the impact (number of reactions received) that the move $M$ has on the dialogue and on the achievement of the current discourse goal and task. If the reaction is positive (the move $\mathrm{M}$ is being assented), then $\mathrm{M}$ receives a positive one $(+1)$ point. If the reaction is negative $(\mathrm{M}$ is not assented $)$ then it receives a negative 0.5 points. The points received by a reaction move depends on the type of learning action underlying the move and take on the default value of the move's impact value. The final value is obtained by the mean value of all moves involved in move $\mathrm{M}$.

The effectiveness value of a move is calculated by the mean value of the number of assent moves received. An assent move $\mathrm{M}$ is identified and recorded after a participant receives $\mathrm{M}$ and consents it. Note that only give-information and raise-an-issue exchange acts can be assented. A negative assent requires a reply move on $M$ to provide further information to reason why $\mathrm{M}$ has not been assented, which generates another move in the current discourse.

Finally, tutor and peer assessment indicators are to evaluate both the quality of the contribution's content by the lecturer monitoring the discussion process and the usefulness of the contribution by the student participating in the discussion. Both indicators are on the scale $0-10$ so as to be accurate in providing mean values of them.

All these quantitative and qualitative indicators are to be weighted adequately according to the specific goals and procedures of each discussion. To that end, a fully customizable environment is necessary to parameterize and adjust each indicator with an appropriate weight by the tutor at any moment of the discussion process.

\section{The development of an effective knowledge-based structured discussion forum}

Based on the conceptual model of interaction management presented in the previous section, a prototype of a web-based collaborative learning system, called Discussion Forum (DF), was developed. This novel experience is reported here from all stages of its development that conducted the design of the prototype that gives new opportunities to learning by discussion, and is applied to meet new pedagogical needs. To this end, a discussion and reasoning process is first described briefly in the form of requirements and then the design of the application is treated in certain detail.

\subsection{Pedagogical background and requirements}

In collaborative learning environments, the discussion process forms an important social task where participants can think about the activity being performed, collaborate with each other through the exchange of ideas arising, propose new resolution mechanisms, and justify and refine their own contributions and thus acquire new knowledge (Salomon, 1993).

To this end, we propose a complete discussion and reasoning process based on three types of generic contributions, namely specification, elaboration and consensus. Specification occurs during the initial stage of the process carried out by the tutor or group coordinator who contributes by defining the group activity and its objectives (i.e. 
Author

statement of the problem) and the way to structure it in sub-activities. Elaboration refers to the contributions of participants (mostly students) in which a proposal, idea or plan to reach a solution is presented. The other participants can elaborate on this proposal through different types of participation such as questions, comments, explanations and agree/disagree statements. Finally, when a correct proposal of solution is achieved, the consensus contributions take part in its approval (this includes different consensus models such as voting); when a solution is accepted the discussion terminates.

Finally, in a discussion process, participants perform a role according to their profile (e.g. coordinator, member, guest, etc.), have personal collaborative preferences (e.g. language) and must set up environment features (e.g. sound or visual effects, text or voice warnings, etc.) according to their personal characteristics. Participant needs are not static and they evolve as the discussion moves forward.

The decision to develop a newly entire system rather than simply adding a module for our purposes to some existing e-learning system came in order to overcome important non-functional requirements arisen in this context, especially in distributed environments. Indeed, non-functional requirements, such as user scalability, resource availability, performance, interoperability, and integration of different, heterogeneous, and legacy collaborative learning systems, may have considerable repercussions on the collaborative learning performance and outcomes when they are not fulfilled appropriately. Failing to meet these important requirements impedes the normal learning flow as well as discriminates learners in terms of technology skills and technical equipment (Caballé, Xhafa, \& Daradoumis, 2007c). For instance, despite having a web-based collaborative learning system with advanced functionalities installed in a server, when the server is down for technical reasons, all participants have to temporarily stop participating, which causes great deal of frustration, especially at our university, where students have very limited time to dedicate to their studies ${ }^{1}$. The lack of existing collaborative learning applications featuring these non-functional aspects encouraged us to entirely build our innovative DF system. Please note that this research line is out of scope in this contribution.

\subsection{The design of the knowledge-based structured discussion forum}

The design of the DF took great advantage of a generic, reusable service-oriented, component-based Collaborative Learning Purpose Library (CLPL) (Caballé, Daradoumis, \& Xhafa, 2007a; Caballé, Daradoumis, \& Xhafa, 2007b) so as to enable a complete and effective reutilization of its generic components for the construction of specific CSCL applications.

The CLPL is made up of five components in all handling user management, security, administration, knowledge management and functionality (see Figure 1). The aim is both to map the essential elements involved in any CSCL collaborative learning application and support the conceptual model of interaction analysis explained in Section 2. To this end, this library is mainly performed by the two components, namely CSCL Knowledge Management and CSCL Functionality components, which form the core of the computational model in the construction of collaborative learning applications. Due to its importance, they are briefly described here (for complete information of the components of the CLPL platform, see (Caballé et al., 2007a):

The CSCL Knowledge Management component is made up of two subsystems, namely CSCL Activity Management and CSCL Knowledge Processing so as to support the first two stages of the information and knowledge management. The first subsystem

\footnotetext{
${ }^{1}$ Students of the Open University of Catalonia are 30 years old on average and $95 \%$ work in full-time jobs.
} 
Fostering collaborative knowledge building by the effective provision of knowledge about the discussion process

manages the system log files made up of all the events occurring in a certain workspace over a given period of time. This event information is then correctly classified according to a complete and tight hierarchy of events based on the mentioned three types of collaborative activity proposed in the previous section. The second subsystem performs the statistical analysis event information as well as the management and maintenance of the knowledge extracted by that analysis.

The CSCL Functionality component, which has five subsystems in all, defines the three elemental parts involved in any form of cooperation, namely coordination, communication and collaboration (Caballé et al., 2007b). Coordination involves the organization of groups to accomplish the important objectives of members such as workspace organization and group structure and planning. Collaboration lets group members share any kind of resources while communication represents the basis of the whole component since it enables coordination and collaboration to be achieved by providing them with low-level communication support. Furthermore, this component implements the presentation to users of the knowledge extracted by the previous component in terms of immediate awareness and constant feedback of what is going on in the system.

To sum up, this platform is used as a computational model especially for both the implementation of the conceptual model of interaction management proposed in Section 2 and the embedding of this information and the knowledge extracted into the discussion process.

The design of the DF includes certain thematic annotation tags based on the lowlevel exchange categories identified in Section 2, such as information-clarification and request of opinion (see Table 1 for a list of all categories), which qualifie each contribution and as a result structure the discussion process. In order to avoid unnecessary choice, each context of the discussion process determines a precise and short list of just those categories that are possible in a certain point of the discussion process (e.g., in replying any kind of request, just the cards involving the provision of information are provided to classify the reply). This makes the choice of the appropriate tag much shorter and easier and no error-prone (see Figure 2). In addition, as part of the design, the tutor is to examine and assess all contributions based partially on the tags used by students to categorize them, and as a result students are aware of the potential repercussions of tagging posts incorrectly in order to optimize the assessment instead of reflecting the true meaning of their posts.

Consequently, all contributions are recorded as exchange acts, analyzed and presented as information to participants either in real time (to guide directly students during the learning activity) or after the task is over (in order to understand the collaborative process). To this end, the CLPL's Knowledge Management component provided full support to the interaction management. In particular, a complete treatment of the structured interaction generated enabled the system to keep participants aware of the contributing behavior and dynamics of others, to check certain argumentative structures during discussion and assist in achieving a more satisfactory solution to the problem during the consensus phase, and finally to provide feedback based on the data produced.

CLPL's Functionality component provided suitable support in the design of the virtual places where the discussions take place. Indeed, the room entity was recursively used in different levels of abstractions, such as folders to hold the assignments featuring the class discussions and discussion threads inside each discussion. This also eased the implementation by reusing the same code for both purposes. This component also provided the suitable means to present the information of the knowledge acquired from the data interaction to the participants in the form of appropriate awareness and feedback, 
Author

representing the cornerstone of this approach. The ultimate aim is to achieve a more effective interaction by allowing all participants to be aware of both their own and others' performance during the discussion process.

Therefore, the DF was especially designed to provide students with additional and important features to support the discussion in comparison to the traditional, well-known discussion tool used in the virtual classrooms of the $\mathrm{UOC}^{1}$, such as (i) updated feedback, which includes the current mean number of all contributions' (see Figure 3 ) and complex indicators about the collaboration (see Figure 4) ${ }^{2}$, (ii) threads in fully separated rooms (see Figure 5), (iii) open-closed branched dialogs (see Figures 6), and (iv) contribution qualifiers (see Figure 2).

Consequently, DF's users were urged to qualify their contributions (using the annotation cards of Table 1) before sending a new or reply post as well as to decide whether their contribution closed the current dialog. In particular, participants' contributions in each thread were designed as structured dialogs with the aim of separating the different types of low-level exchanges. Moreover, dialogs are to be closed when a request is satisfied or a basic problem is and to be branched from a specific exchange (i.e. problem-statement) so as to provide different solutions to the same statement (see Figure 6). Finally, a contribution is both to be assented depending on the context and to be evaluated by the other participants in terms of utility in their progress in the discussion (see Figure 7).

A discussion process is conducted by a tutor who continuously monitors the discussion threads with the aim of both assessing the contributions and providing support when needed by posting clarifying contributions in any thread and/or starting supporting threads. The contribution assessment by the tutors is performed in a very similar way as the peer assessment (see Figure 7) and becomes very smoothly even in the case of largesize groups. This is achieved by first selecting the suitable mark of each contribution in a discussion thread and then by performing a single submission to assess all the contributions in the thread.

Finally, for the sake of a rapid prompt of the awareness and feedback information to students and tutors, our research group have successfully managed to embed this information into the group activity in an efficient manner, even in real time. Indeed, our experience at the UOC has shown the need to monitor and evaluate real, long-term, complex, collaborative problem-solving situations. As a result, there is a strong need for powerful tools that record the large volume of interaction data generated during the group activity and can then be used to perform an efficient interaction analysis and knowledge extraction. Given the real needs of any online collaborative learning situation, in order to provide different types of awareness and feedback, we need to capture all and each type of possible data that could result to a huge amount of information that is generated and gathered in data log files. Moreover, the need to make the analyzed information available in real time entails that we may come across with processing requirements beyond those of a single computer. To this end, several studies (for a detailed description of these

\footnotetext{
${ }^{1}$ Since the UOC started in 1994, its virtual classrooms include a typical and limited bulletin board system (BBS) to support class assignments based on asynchronous discussion, where students and tutors post, reply, and read messages. Despite the system provides certain important functionalities, (e.g., discussion threads), neither they are well-accomplished nor other essential features are provided, such as the provision of feedback information about what is going on during the group activity. This lack impedes fundamental aspects of the collaborative learning process to occur, such as students' self-regulation and scaffolding, as well as tutors' monitoring tasks, which become very tedious since they must be performed manually.

2 Please note that in Spain it is allowed to publish the students' names and their partial grades (i.e., peer and tutor assessment). In the case of those countries whose University policies would bring a serious bridge of privacy laws when publishing this information, the name of students could be easily replaced by anonymous identifiers without losing any positive effect from the feedback provided.
} 
Fostering collaborative knowledge building by the effective provision of knowledge about the discussion process

studies, see Caballé, Xhafa, \& Daradoumis, 2008) have been conducted to show how a parallel approach can increase the efficiency of processing a large amount of interaction data and achieve an effective embedding of the appropriate knowledge extracted into collaborative learning practices.

The tutor assessment, along with the rest of indicators presented, are automatically and constantly processed by the system. For monitoring purposes, the system proposes an updated final mark of the progress of each student based on all the indicators presented (see Figure 8). Last column in Figure 8 shows a numeric mark on the scale $0-10$ for each student automatically generated and updated by the system. This final mark is based on all the indicators presented, which are adjusted with different weights. For the purpose of this specific discussion, the weights were set as follows: activity: $10 \%$; passivity: $10 \%$; impact: $20 \%$; effectiveness: $10 \%$; assessment: $50 \%$. Please note that this information including the students' final mark is provided to the tutor only since in Spain it is not allowed to publish the students' final grades though it is allowed to publish both the names of students and non-conclusive assessment (i.e. quality assessment). These indicators are to be adjusted with appropriate weights by the tutor so as to reinforce certain aspects of the discussion process according to the specific pedagogical objectives of the learning task.

\section{Results and discussion on the effects in the learning experience}

In order to evaluate the prototype of the DF and analyze its effects in the discussion process, 80 graduated students enrolled in the course Methodology and Management of Computer Science Projects during the last term were involved in this experience. Students were equally distributed into two classrooms and participated in the experience at the same time. Students from one classroom were required to use the standard asynchronous threaded discussion forum offered by the virtual campus of the UOC while the other group of students used the new DF outside the virtual campus to support the same discussions with the same rules during the same time (i.e., five weeks in all).

The whole experience consisted in two discussion assignments separated in time with very different goals and procedures so as to validate the flexibility of the approach. The first assignment in both groups lasted two weeks and consisted of discussing the same issue: project management requirements vs. product requirements. In this assignment, each student was required to start a discussion thread with posting a contribution on the issue in hand, which resulted in as many threads as students. At the end of the discussion, each student was asked to close his/her thread with an improved contribution on the issue according to what s/he had learnt in the discussion. During the discussion, any student could contribute in both the own and any other discussion thread as many times as needed, as well as start extra threads to provide new argumentations or approaches with regards to the issue addressed. The aim was to evaluate the effect of the discussion process in the acquisition of knowledge of each student by comparing the quality of each thread's first and last contribution posted by the same student.

A statistical analysis of the results in the first discussion comparing both the standard and the DF tools is shown in Table 2. Despite the standard tool generated more threads, most of them were actually empty (i.e. just 8 threads were contributed with more than 1 post vs. 42 threads in the DF). Moreover, the SD statistic for the posts/thread mean appears to be high in the DF, which proves the heterogeneity of the discussion involving threads of very different length. Note the very high SD statistic in the posts/students mean due to a single outlier, without which SD is 6,3. Finally, quality statistics are shown in terms of the number of words per contribution and the tutor assessment on the content. 
Author

The higher number of number of words in the standards tool is due to the lack of discussion as most of threads were just started with a long opening contribution as a problem statement. On the other hand, the DF generated actual discussion and as a result the contributions became highly structured and specific. The tutor assessment row refers to content quality of all the contributions on average.

The qualitative evaluation of this first discussion was addressed by both examining those discussion threads that contained enough discussion (i.e. more than 7 posts) and checking whether the student who was in charge of each thread had posted both a start and close contribution on the same issue. The results on the DF showed that, in 28 threads fulfilling these requirements, $32 \%$ of students had improved their qualitative mark through the discussion in their threads, $68 \%$ kept the same mark, and no mark had dropped. On the other hand, no results were extracted from the discussion using the standard tool as it was poorly contributed; just 8 threads showed some discussion but only 4 had more than 7 posts.

The second assignment in both groups was held at the end of the same academic term, one month after finishing the previous one and lasted for three weeks. It consisted of discussing the stage of closing a software project. The procedure was the following: students were free to open zero, one or several discussion threads where they proposed specific objectives, activities, and processes needed to appropriately close a software project. Hence, in this discussion there was no requirement to open a discussion thread and all students could participate in the discussion threads at convenience. At the end of the discussion, those students who had opened a discussion thread were asked to close it by sending a contribution that summarized and concluded the main points arisen in the thread.

The statistical analysis of the results extracted from the second discussion comparing both the standard and the DF tools are shown in Table 3. In comparison to the previous assignment, there is a decrease in the number of contributions in both groups, which may be explained by two reasons: even though the number of potential participants was the same as the previous discussion, $40 \%{ }^{1}$ in each group had already made the decision to give up the course before this second discussion started and as a result most of them did not pay attention nor contribute to the discussion. Moreover, the participation was not a requirement in this assignment and consequently some students chose not to participate.

The qualitative evaluation of the second discussion was addressed in a similar way as the previous one. Despite the standard tool rated high in the number of threads, just one of them provided real discussion (i.e., more than 7 posts). Hence, it could be stated that no discussion was achieved using the standard tool. On the other hand, the DF performed much better providing actual discussion in 16 out of 21 threads achieved.

The mean number of words per contribution in the standard tool also rated higher than the DF in this second experience. This confirms the effects of the inherent structure and richness provided to the discussion process by the DF whereas the standard tool promotes large monolithic one-sided points of view. Finally, the standard tool achieved a higher average mark on the qualitative content of the contributions. It could be argued that most of participants of the standard tool were good students, whose first and only contribution to a thread was fine. However, the lack of discussion missed many important aspects, such as reactive participation behavior and peer involvement skills, which are fundamental to achieve a successful discussion process. All these aspects are to be combined with the evaluation of the qualitative content to form the final assessment of the collaborative learning activity.

\footnotetext{
${ }^{1}$ Currently, the drop-out average at the Open University of Catalonia is about $50 \%$.
} 
Fostering collaborative knowledge building by the effective provision of knowledge about the discussion process

Table 4 shows the results of a structured and qualitative report conducted at the end of the discussions addressed to the DF' users who were also asked to compare it to the standard well-known tool they had already used in previous courses at the UOC.

Finally, in order to evaluate the reliability of the automatic assessment approach in both assignments, the tutor supervising the discussions supported by the DF was required to both (1) submit a precise assessment on content quality of every contribution posted, which was presented to students as feedback information (see subsection 2.1. and Figure 4 for further information on tutor assessment) and (2) evaluate students' performance manually by the tutor by filling out a spreadsheet that helped score each student's participation according to both the content quality of each of his/her contribution and the purpose and context where the contribution took place (e.g., whether it was a new argumentation or a reply, brought interesting opportunities for further discussion, it was just a greeting-type post, etc.). This second evaluation task could be complemented with extra information on individual behavior in the discussion added by the tutor according to his knowledge and experience in this type of class assignment.

The ultimate aim of this double evaluation process was to compare the manual evaluation performed by the tutor to the semi-automatic assessment process provided by the system. To this end, each evaluation process resulted in proposing both a final mark for each student and a position list where all students were ranked according to his/her final mark (see first and last columns in the feedback information depicted in Figure 8). In the semi-automatic evaluation, on the one hand, the system addressed four indicators, namely, activity, passivity, impact and effectiveness, becoming $50 \%$ of automatic evaluation. The rest of the evaluation came from the quality indicator only, which was addressed by the tutor who was in charge of assessing the contributions' content quality $(40 \%)$, and the peers who assessed the usefulness of others' contributions on average (see also Figure 8 for further information). Please note that these percentages may vary according to the type of the discussion and they can be adjusted by the tutor. On the other hand, the manual evaluation process was carried out entirely by the tutor and followed the same assessment procedure as that performed while using the standard discussion tool of the UOC.

The results of the automatic assessment were very promising since the tutor in charge of the DF agreed with the final marks proposed by the system in more than $75 \%$ of cases. 31 out of 40 students in the DF's rank matched the same position as in the rank appeared in the tutor's spreadsheet. In addition, the tutor reported the promising benefits from the DF in the monitoring process on the discussion since this new tool alleviates tutors and moderators from the tedious work of tracking and evaluating the discussion's dynamics and outcomes manually. On the other hand, a clear inconsistency was identified since all final marks proposed by the system scored 1.1 points on average lower that those proposed by the tutor, thus showing the need to weight the indicators in the DF more objectively. In overall, these results are not conclusive but they encourage us to undertake more experimentation and especially validation processes on the automatic assessment approach.

\section{Conclusions and future work}

This paper describes a promising approach for enhancing knowledge management that contributes to the improvement of the discussion process in virtual collaborative learning environments. To this end, the experience of an innovative Discussion Forum has been reported. The results of this experience are not conclusive due to its exploratory nature. However, from the analysis of the results it has been proved to promise significant benefits for students in the context of project-based learning, and in education in general. 
Author

A decentralized distributed infrastructure has been recently added to the DF prototype in order to meet certain important non-functional requirements that may influence the learning process a great deal (Caballé et al., 2007c), such as performance, scalability, fault-tolerance, and interoperability. The gain in performance might help us, for instance, include more complex information of the collaboration to be generated and presented in real time (such as modeling the participants' behavior during the discussion by combining individual and group session and navigation information). Moreover, the benefits from having a flexible, interoperable environment makes it possible to completely integrate the DF system into the UOC's virtual campus. The aim of these improvements is both to enhance the effectiveness of complex collaborative learning processes (e.g., by avoiding a central point of failure) and stimulate the learning experience by describing and predicting students' actions and intentions as well as adapting the learning process to students' features, habits, interests, preferences, and so on. We plan to explore these interesting possibilities in the next iterations of the DF design.

\section{Acknowledgments}

This work has been partially supported by the Spanish MCYT project TSI2005-08225C07-05.

\section{References}

Bratitsis, T. \& Dimitracopoulou, A. (2006). Monitoring and Analysing Group Interactions in asynchronous discussions with the DIAS system. Lecture Notes in Computer Science, LNCS 4154. (pp. 54-61). Heidelberg-Berlin: Springer-Verlag. ISBN: 978-3-540-39591-1.

Caballé, S., Xhafa, F., \& Daradoumis, T. (2008). A Grid Approach to Efficiently Embed Information and Knowledge about Group Activity into Collaborative Learning Applications. The Learning Grid Handbook. Chp. 11 (pp. 166-190). Amsterdam: IOS Press (in press).

Caballé, S., Daradoumis, T., \& Xhafa, F. (2007a). A Generic Platform for the Systematic Construction of Knowledge-based Collaborative Learning Applications. Architecture Solutions for e-Learning Systems. Idea Group Inc (IGI). Chp. XII (pp. 219-242). Press, Hershey, PA: Idea Group, ISBN: 978-1-59904-633-4.

Caballé, S., Daradoumis, T., \& Xhafa, F. (2007b). Efficient Embedding of Information and Knowledge into CSCL Applications. Lecture Notes in Computer Science, LNCS 4469. (pp. 548-559). Heidelberg-Berlin: Springer-Verlag. ISBN: 978-3-540-73010-1.

Caballé, S., Xhafa, F., \& Daradoumis, T. (2007c). A Service-oriented Platform for the Enhancement and Effectiveness of the Collaborative Learning Process in Distributed Environments. Lecture Notes in Computer Science, LNCS 4804. (pp. 1280-1287). Heidelberg-Berlin: Springer-Verlag. ISBN: 978-3-540-76835-7.

Clark, H. \& Schaefer, E. (1989). Contributing to discourse. Cognitive Science. 13(2), 259-294.

Daradoumis, T., Martínez, A., \& Xhafa, F. (2006). A Layered Framework for Evaluating Online Collaborative Learning Interactions. International Journal of HumanComputer Studies. Special Issue on "Theoretical and Empirical Advances in Groupware Research",Volume 64, Issue 7 (pp. 622-635). Academic Press: Elsevier Ltd, ISSN: 1071-5819, July 2006

De Smet, M., Van Keera, H. \& Valckea, M. (2008). Blending asynchronous discussion groups and peer tutoring in higher education: An exploratory study of online peer tutoring behaviour. Computers \& Education. 50(1): 207-223, Academic Press: Elsevier Ltd, January 2008. 
Fostering collaborative knowledge building by the effective provision of knowledge about the discussion process

De Wever, B., Schellens, T., Valcke, M., \& Van Keer, H. (2006). Content analysis schemes to analyze transcripts of online asynchronous discussion groups: A review. Computers \& Education. 46(1): 6-28, Academic Press: Elsevier Ltd, January 2006.

Dillenbourg, P. (1999). Introduction; What do you mean by "Collaborative Learning”? In P. Dillenbourg (Ed.), Collaborative learning. Cognitive and computational approaches. (pp. 1-19). Oxford: Elsevier Science.

Gutwin, C., Stark, G., \& Greenberg, S. (1995). Support for Workspace Awareness in Educational Groupware. Proceedings of the ACM Conference on Computer Supported Collaborative Learning, Bloomington, Indiana, USA.

Hew, K.F. \& Cheung, W.S. (2008). Attracting student participation in asynchronous online discussions: A case study of peer facilitation. Computers \& Education. Academic Press: Elsevier Ltd, (in press).

Martin, J.R. (1992). English Text: Systems and Structure. Amsterdam: Benjamin Press.

McGrath, J.E. (1991). Time, Interaction and Performance (TIP). A Theory of Groups. Small Group Research, 22: 147-174.

Pena-Shaff, J. \& Nicholls, C. (2004). Analyzing student interactions and meaning construction in computer bulletin board discussions. Computers \& Education. 42(3): 243-265, Academic Press: Elsevier Ltd, April 2004.

Pilkington, R.M. (1999). Analysing Educational Discourse: The DISCOUNT Scheme. Technical Report No. 99/2, Jan 1999, The University of Leeds, Leeds, England.

Puntambekar, S. (2006). Analyzing collaborative interactions: divergence, shared understanding and construction of knowledge. Computers \& Education. 47(3): 332351, Academic Press: Elsevier Ltd, November 2006.

Salomon, G. (1993). No distribution without individual's cognition: a dynamic interactional view. In G. Solomon (Ed.), Distributed cognitions (pp. 111-138). Cambridge, MA: Cambridge University Press.

Schellens, T. \& Valcke, M. (2006). Fostering knowledge construction in university students through asynchronous discussion groups. Computers \& Education. 46(4):349-370, Academic Press: Elsevier Ltd, May 2006.

Schrire, S. (2006). Knowledge building in asynchronous discussion groups: Going beyond quantitative analysis. Computers \& Education. 46(1): 49-70, Academic Press: Elsevier Ltd, January 2006.

Schwartz, D. L. (1999). The productive agency that drives collaborative learning. In: P. Dillenbourg (Ed.), Collaborative learning: Cognitive and computational approaches. (pp. 197-219). NY: Elsevier Science/Permagon.

Self, J.A. (1994). Dormobilea: vehicle for metacognition. In: Chan, T.W. and Self, J.A. (Eds.), Emerging Computer Technologies in Education, (pp. 1-20). Charlottesville, VA: AACE.

Soller, A. (2001). Supporting Social Interaction in an Intelligent Collaborative Learning System. Int. J. of Artificial Intelligence in Education, 12: 40-62.

Stahl, G. (2006). Group Cognition: Computer Support for Building Collaborative Knowledge. Acting with Technology Series, Cambridge, MA: MIT Press. ISBN: 978-0262195393.

Strijbos, J-W., Martens, R., Prins, F., \& Jochems, W. (2006). Content analysis: What are they talking about? Computers \& Education. 46(1): 29-48, Academic Press: Elsevier Ltd, January 2006.

Zumbach, J., Hillers, A., \& Reimann, P. (2003). Supporting Distributed Problem-Based Learning: The Use of Feedback in Online Learning. In T. Roberts (Ed.), Online Collaborative Learning: Theory and Practice (pp. 86-103). Hershey, PA: Idea Group. 
Author

\begin{tabular}{|l|l|}
\hline \multicolumn{1}{|c|}{ Exchange moves } & \multicolumn{1}{|c|}{ Exchange categories } \\
\hline \multirow{5}{*}{ support } & Greeting \\
\cline { 2 - 2 } & Encouragement \\
\cline { 2 - 2 } & Motivation \\
\cline { 2 - 2 } & REQUEST-Information \\
\cline { 2 - 2 } & REQUEST -Elaboration \\
\cline { 2 - 2 } & REQUEST -Clarification \\
\cline { 2 - 2 } & REQUEST -Justification \\
\cline { 2 - 2 } & REQUEST -Opinion \\
\cline { 2 - 2 } & REQUEST -Illustration \\
\hline \multirow{5}{*}{ inform } & INFORM-Extend \\
\cline { 2 - 2 } & INFORM-Lead \\
\cline { 2 - 2 } & INFORM-Suggest \\
\cline { 2 - 2 } & INFORM-Elaboration \\
\cline { 2 - 2 } & INFORM-Explain/Clarification \\
\cline { 2 - 2 } & INFORM-Justify \\
\cline { 2 - 2 } & INFORM-State \\
\cline { 2 - 2 } & INFORM-Agree \\
\cline { 2 - 2 } & INFORM-Disagree \\
\hline set-up-an-issue & PROBLEM-Statement \\
\hline provide-solution & PROBLEM-Solution \\
\hline consent-solution & PROBLEM-Extend solution \\
\cline { 2 - 2 } & PROBLEM-Assent solution \\
\hline
\end{tabular}

Table 1. List of the exchange moves and exchange categories to classify a contribution. 
Fostering collaborative knowledge building by the effective provision of knowledge about the discussion process

\begin{tabular}{|l|c|c|}
\hline \multicolumn{1}{|c|}{ Statistics } & Standard tool & $\mathrm{DF}$ \\
\hline Number of students & 40 & 40 \\
\hline Number of threads & 48 & 44 \\
\hline Total of posts & 95 & $\mathrm{M}=7,9 \mathrm{SD}=5,0$ \\
\hline $\begin{array}{l}\text { Mean number } \\
\text { (posts/thread) }\end{array}$ & $\mathrm{M}=1,9 \mathrm{SD}=2,4$ & $\mathrm{M}=8,7 \mathrm{SD}=8,1$ \\
\hline $\begin{array}{l}\text { Mean number } \\
\text { (posts/student) }\end{array}$ & $\mathrm{M}=2,3 \mathrm{SD}=1,9$ & $\mathrm{M}=286 \mathrm{SD}=85$ \\
\hline $\begin{array}{l}\text { Mean number } \\
\text { (words/contribution) }\end{array}$ & $\mathrm{M}=352 \mathrm{SD}=139$ & 7.6 \\
\hline $\begin{array}{l}\text { Tutor assessment (average, } \\
\text { out of 10) }\end{array}$ & 7.2 & \\
\hline
\end{tabular}

Table 2. Main statistics extracted from the first class assignment using both discussion tools. 
Author

\begin{tabular}{|l|c|c|}
\hline \multicolumn{1}{|c|}{ Statistics } & Standard tool & DF \\
\hline Number of students & 40 & 40 \\
\hline Number of threads & 43 & 21 \\
\hline Total of posts & 71 & 199 \\
\hline $\begin{array}{l}\text { Mean number } \\
\text { (posts/thread) }\end{array}$ & $\mathrm{M}=1,6 \mathrm{SD}=0,4$ & $\mathrm{M}=9,4 \mathrm{SD}=3,2$ \\
\hline $\begin{array}{l}\text { Mean number } \\
\text { (posts/student) }\end{array}$ & $\mathrm{M}=421 \mathrm{SD}=1,1$ & $\mathrm{M}=4,9 \mathrm{SD}=4,1$ \\
\hline $\begin{array}{l}\text { Mean number } \\
\text { (words/contribution) }\end{array}$ & $\mathrm{M}=310 \mathrm{SD}=85$ \\
\hline $\begin{array}{l}\text { Tutor assessment (average } \\
\text { out of 10) }\end{array}$ & 8,1 & 7,5 \\
\hline
\end{tabular}

Table 3. Main statistics of the second class assignemnt using both discussion tools. 
Fostering collaborative knowledge building by the effective provision of knowledge about the discussion process

\begin{tabular}{|c|c|c|}
\hline Selected questions & $\begin{array}{l}\text { Average of } \\
\text { structured } \\
\text { responses } \\
(0-5)\end{array}$ & $\begin{array}{c}\text { Excerpt of } \\
\text { students' comments }\end{array}$ \\
\hline $\begin{array}{l}\text { Asses the Discussion Forum } \\
\text { (DF) }\end{array}$ & 3 & \multirow{4}{*}{$\begin{array}{l}\text { "Apart from some technical problems, } \\
\text { the DF fulfilled my expectations" } \\
\text { "The statistical data and quality } \\
\text { assessment displayed influenced my } \\
\text { participation" } \\
\text { "The standard tool is a chaos for large } \\
\text { debates (...) DF's discussion rooms } \\
\text { eased me the debate a lot" } \\
\text { "The DF should be used to support } \\
\text { debates in other courses" }\end{array}$} \\
\hline $\begin{array}{l}\text { Evaluate how the DF fostered } \\
\text { your active participation }\end{array}$ & 4 & \\
\hline $\begin{array}{l}\text { Did the DF help you acquire } \\
\text { knowledge on the debate's } \\
\text { issue? }\end{array}$ & 4 & \\
\hline $\begin{array}{l}\text { Compare the DF to the } \\
\text { campus' standard forum tool }\end{array}$ & 4 & \\
\hline
\end{tabular}

Table 4. Excerpt of a questionnaire's results on both the DF and the standard tool to support the discussion process. 
Author

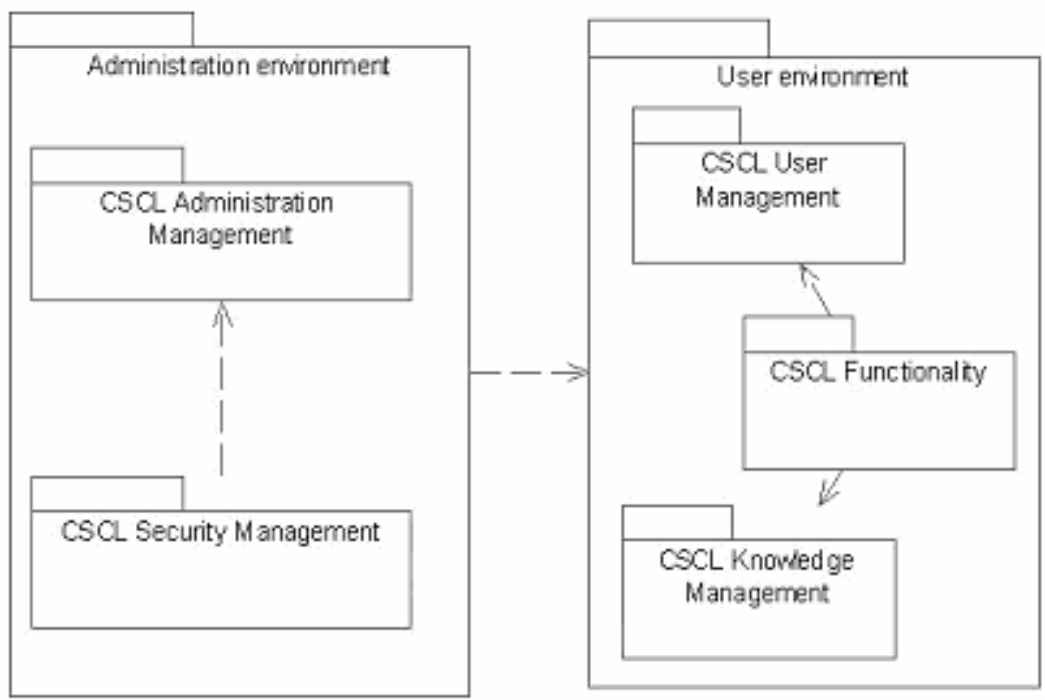

Figure 1. Graphical representation of the components of the CLPL platform and their dependencies. 
Fostering collaborative knowledge building by the effective provision of knowledge about the discussion process

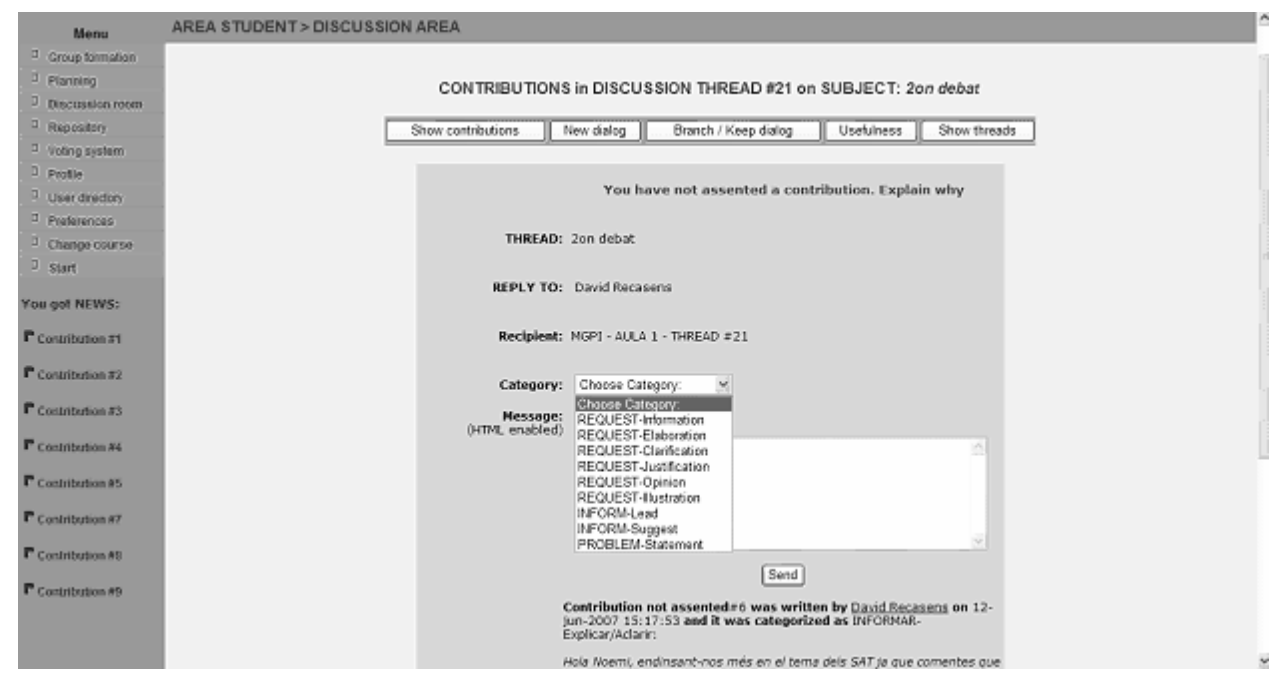

Figure 2. The specific list of cards for a reply to a contribution categorized as INFORM-Explain. 


\section{Author}

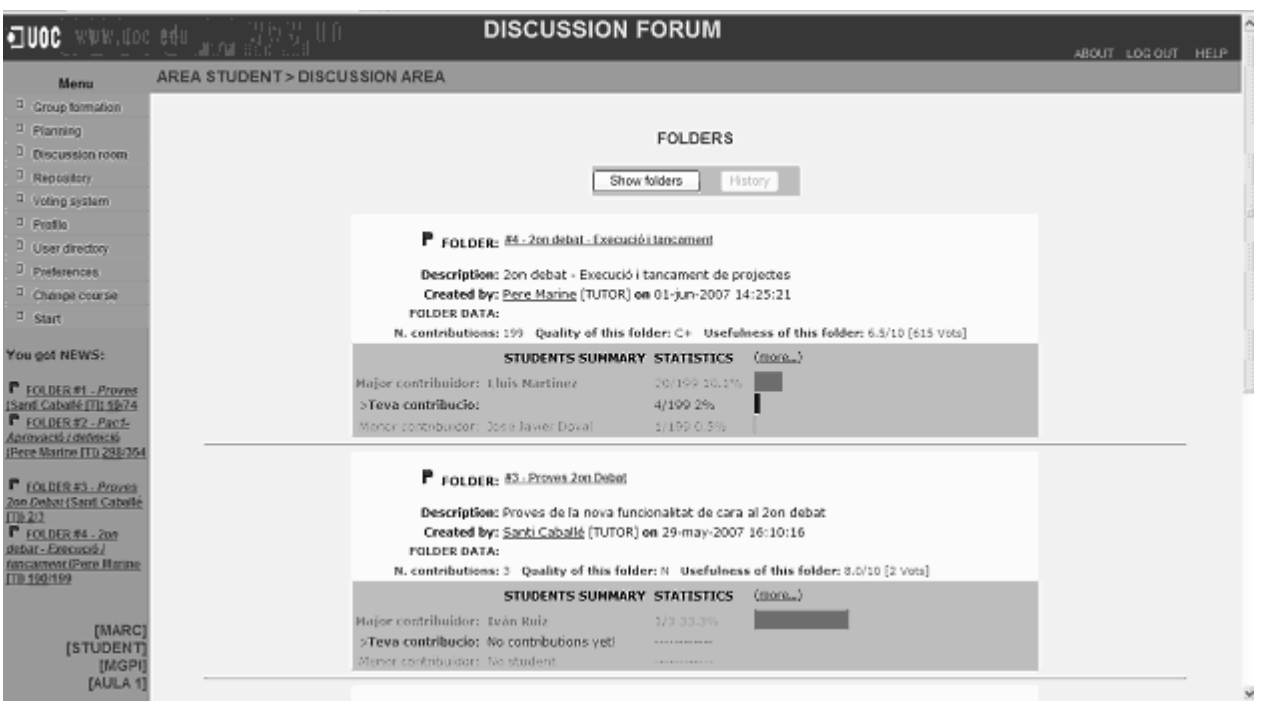

Figure 3. A snapshot of awareness and simple quantitative feedback provided. 
Fostering collaborative knowledge building by the effective provision of knowledge about the discussion process

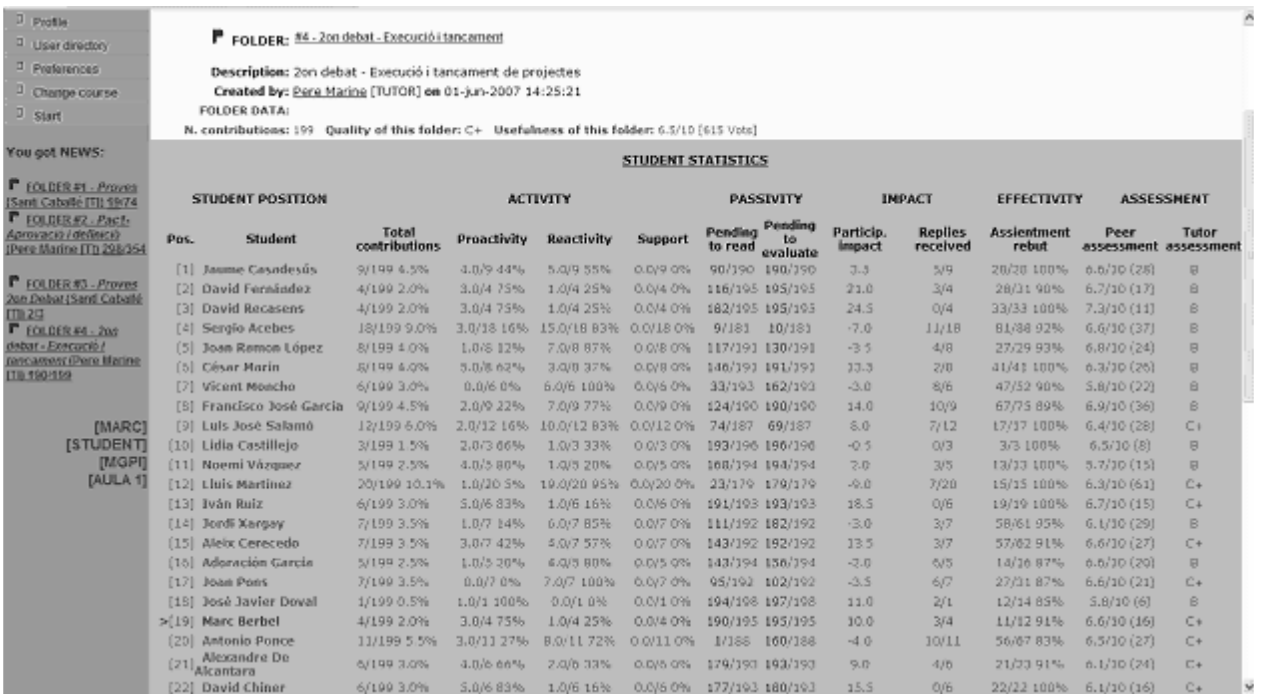

Figure 4. A snapshot of complex and updated feedback provided to all participants. In this case, student Marc is located in the 19th position in the rank. 


\section{Author}

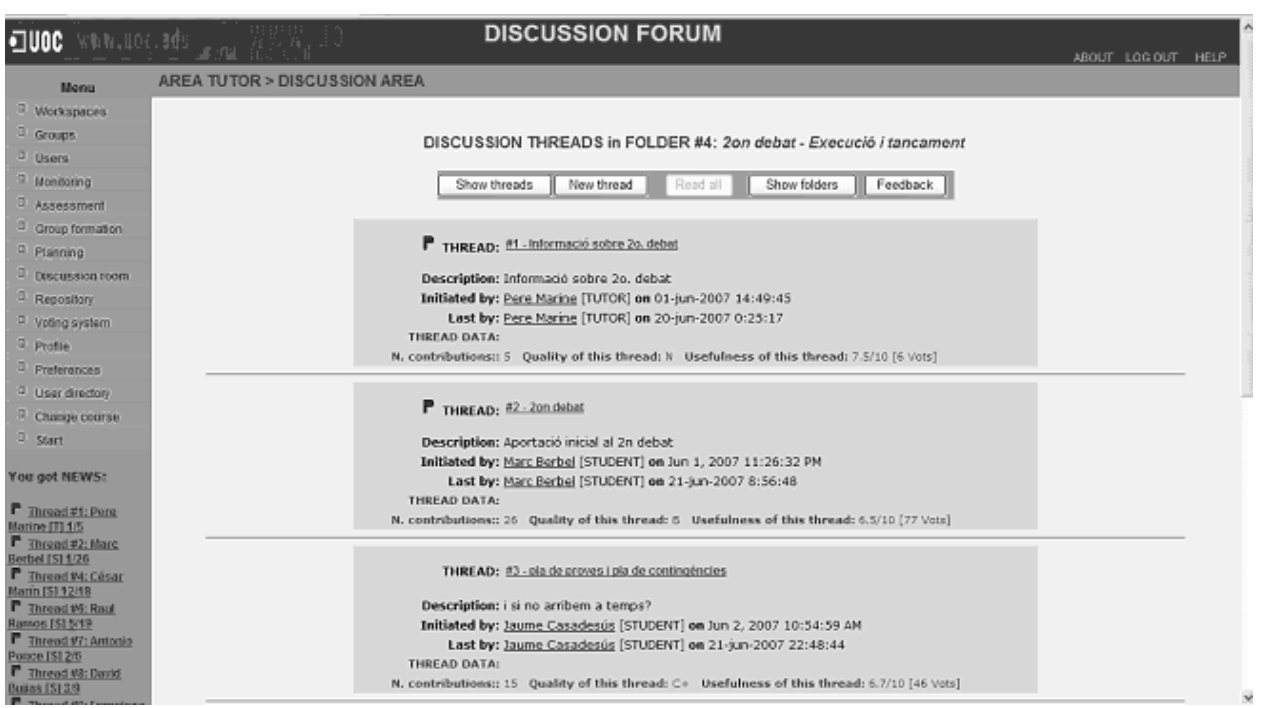

Figure 5. A snapshot of some discussion threads inside a folder holding the discussion. 
Fostering collaborative knowledge building by the effective provision of knowledge about the discussion process
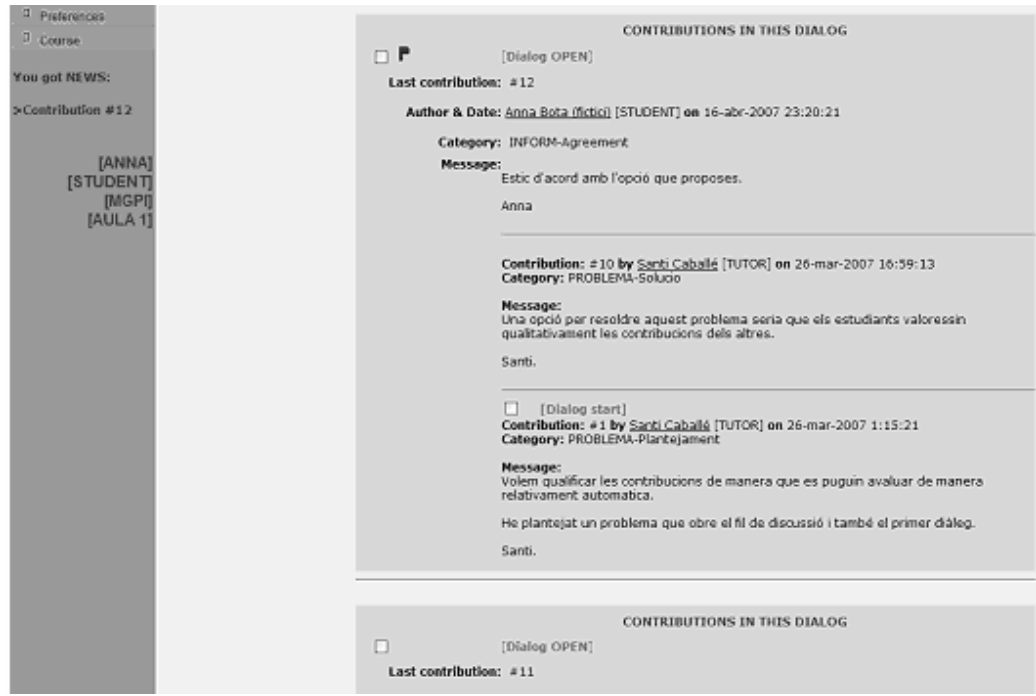

Figure 6. Two dialogs in the same thread; upper dialog can be branched by checking the first checkbox. 
Author

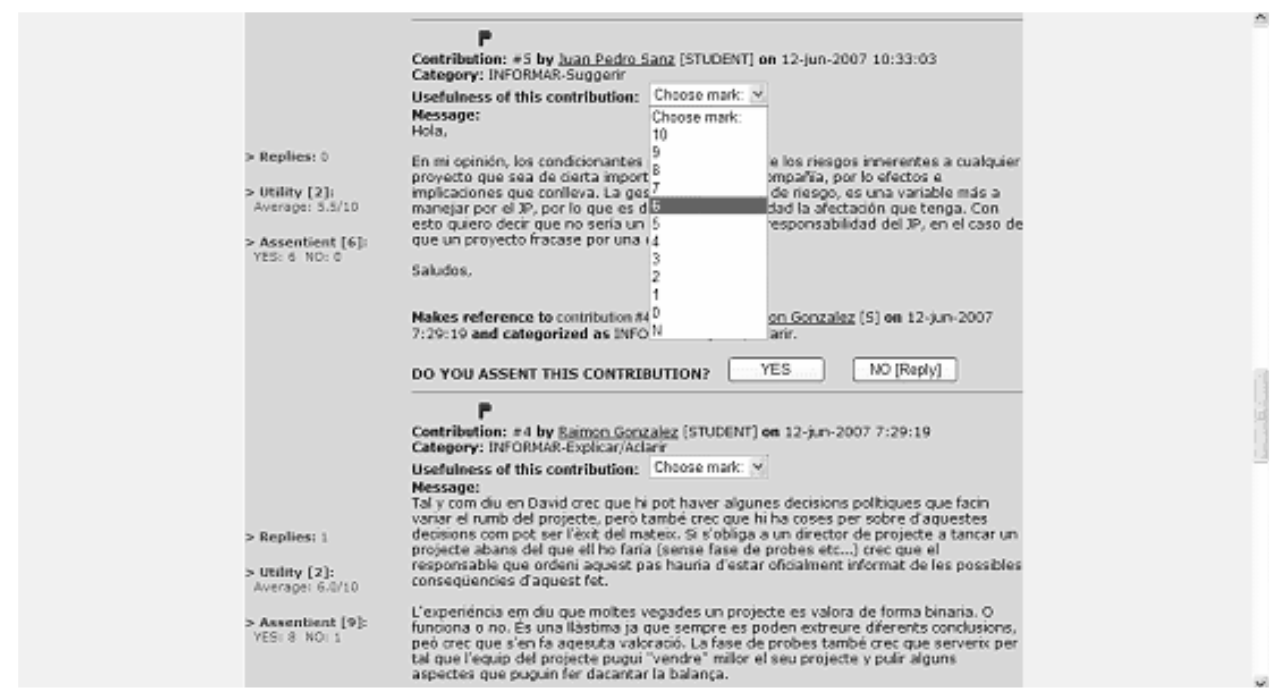

Figure 7. An example of a contribution to be assented and evaluated by a participant. 
Fostering collaborative knowledge building by the effective provision of knowledge about the discussion process

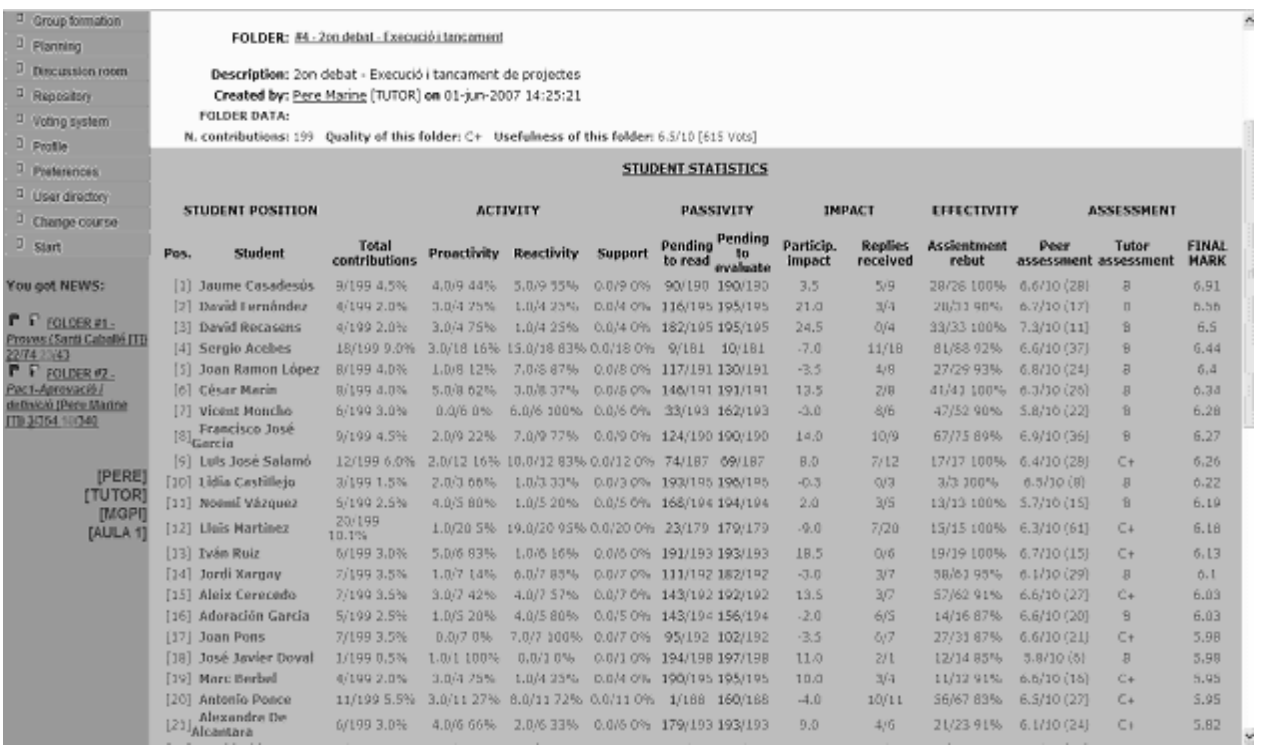

Figure 8. Monitoring information provided to the tutor. 